\title{
THE CULTURAL EVOLUTION OF SPATIAL MODULATIONS IN ARTIFICIAL SIGN LANGUAGES
}

\author{
YASAMIN MOTAMEDI*1, MARIEKE SCHOUWSTRA ${ }^{2}$, JENNIFER \\ CULBERTSON $^{2}$, KENNY SMITH ${ }^{2}$, SIMON KIRBY ${ }^{2}$ \\ *Corresponding Author: y.motamedi@ucl.ac.uk \\ ${ }^{1}$ Experimental Psychology, University College London, London, UK \\ ${ }^{2}$ Centre for Language Evolution, University of Edinburgh, Edinburgh, UK
}

Sign languages use a range of linguistic tools to denote the relationship between a predicate and its arguments, some which are shared with spoken languages (e.g. lexical contrasts and word order). However, many sign languages also make use of modality-specific spatial modulations to denote who does what to whom. The most common manifestation cross-linguistically of such spatial modulations is referential indexing, where animate arguments are indexed with a location in space and those indexed locations are referred to over a stretch of discourse to refer to the same argument. A characteristic example of this is given in 1); the man and the woman are represented by indexed locations - $a$ and $b$, respectively — and the verb ask moves between the referenced locations.

1. MAN $_{\mathrm{a}} \mathrm{WOMAN}_{\mathrm{b}} \mathrm{ASK}_{\mathrm{b}}$

'The man asks the woman'

Though spatial modulation of this kind is widespread cross-linguistically, and has been considered near-universal in sign languages, recent study of emerging sign languages suggests that systematic spatial reference does not emerge fully formed but emerges and systematizes over generations of a new language (Padden et al., 2010; Kocab et al., 2014). We set out to examine how a tool that relies heavily on the iconic use of space systematizes over time, and whether an understanding of this process can shed light on the debate over the linguistic nature of spatial modulations (Cormier et al., 2015; Liddell, 2003; Lillo-Martin and Meier, 1999).

We present an experimental investigation into the emergence and evolution of spatial modulations in the manual modality, asking participants to communicate 
about a set of events involving one or more animate arguments. The experiment took the form of a silent gesture task, in which hearing participants with no knowledge of sign language had to communicate a set of events using only gesture. The task combined both interaction, in the form of a director-matcher task, and iteration, with pairs of participants organized in transmission chains. A pair of participants was first trained on a set of gestures produced by a participant in the previous generation, and then in testing had to communicate about the same events, taking turns to produce (direct) and interpret (match) a gesture. The gestures they produced were then transmitted as training for another pair. The first pair in each chain received no training, but had to innovate gestures for the events. The events participants communicated about were presented as pairs of English sentences, containing one or both of the arguments Hannah and Sarah. Sentence pairs were either same-agent (where the agent in both sentences is the same), or different-agent (where the agent is different in sentence 1 and 2). Sentence pairs were presented within blocks of 4 , with participants switching director/matcher roles after each block, and each block presenting either same-agent or differentagent sentence pairs. Within a block, each sentence pair demonstrated one of four verb types: verbs of motion (no endpoint), verbs of motion (endpoint), verbs of physical transfer, and verbs of non-physical transfer. The block structure presents a 'discourse' over which participants must repeatedly refer to Hannah and Sarah and differentiate between them to successfully communicate.

Gestures were coded on several parameters: type of gesture for agent and verb, location of agent and verb gesture, and path of verb gesture. Our results showed that the use of these parameters to disambiguate arguments in target sentences can be grouped into three main strategies: 1) a lexical strategy, in which participants vary the type of gesture used to denote the agent, 2) a body strategy in which body orientation (mapping onto verb location and path) signal the agent, and 3) the indexing strategy, in which participants index locations in the space around them (varying agent location). Over generations in the experiment, participants converge on a particular strategy, where it becomes further systematised. The strategies participants use reflect iconic strategies that find analogues in natural sign languages (e.g. lexical signs, body shift, and agent marking). Participants innovate highly iconic strategies that are systematized over generations in transmission chains. The evolution of participants' gestures reflects changes in emerging sign languages, showing a development from body-centered movement paths to gesture paths abstracted away from the body (Padden et al., 2010), and support experimental findings demonstrating the evolution of linguistic structure through cultural evolutionary processes (Kirby et al., 2015). 


\section{References}

Cormier, K., Fenlon, J., \& Schembri, A. (2015). Indicating verbs in British Sign Language favour motivated use of space. Open Linguistics, 1, 684-707.

Kirby, S., Tamariz, M., Cornish, H., \& Smith, K. (2015). Compression and Communication in the Cultural Evolution of Linguistic Structure linguistic structure. Cognition, 141, 87-102.

Kocab, A., Pyers, J., \& Senghas, A. (2014). Referential Shift In Nicaraguan Sign Language: A Transition From Lexical To Spatial Devices. Frontiers in Psychology

Liddell, S. K. (2003). Grammar, gesture and meaning in American Sign Language. Cambridge: Cambridge University Press.

Lillo-Martin, D., \& Meier, R. P. (2011). On the linguistic status of "agreement" in sign languages. Theoretical Linguistics, 37(3-4), 95-141.

Padden, C., Meir, I., Aronoff, M., \& Sandler, W. (2010). The grammar of space in two new sign languages. Sign Languages: A Cambridge Language Survey. Cambridge University Press, Cambridge, UK, 570-592. 